\title{
Effect of overexpression of SNF1 on the transcriptional and metabolic landscape of baker's yeast under freezing stress
}

\author{
Lu Meng ${ }^{1}$, Xu Yang ${ }^{1}$, Xue Lin ${ }^{1,2,3^{*}} \mathbb{D}$, Huan-Yuan Jiang ${ }^{1}$, Xiao-Ping Hu${ }^{1,2,3}$ and Si-Xin Liu ${ }^{4}$
}

\begin{abstract}
Background: Freezing stress is the key factor that affecting the cell activity and fermentation performance of baker's yeast in frozen dough production. Generally, cells protect themselves from injury and maintain metabolism by regulating gene expression and modulating metabolic patterns in stresses. The Snf1 protein kinase is an important regulator of yeast in response to stresses. In this study, we aim to study the role of the catalytic subunit of Snf1 protein kinase in the cell tolerance and dough leavening ability of baker's yeast during freezing. Furthermore, the effects of SNF1 overexpression on the global gene expression and metabolite profile of baker's yeast before and after freezing were analysed using RNA-sequencing and untargeted UPLC - QTOF-MS/MS, respectively.

Results: The results suggest that overexpression of SNF1 was effective in enhancing the cell tolerance and fermentation capacity of baker's yeast in freezing, which may be related to the upregulated proteasome, altered metabolism of carbon sources and protectant molecules, and changed cell membrane components. SNF1 overexpression altered the level of leucin, proline, serine, isoleucine, arginine, homocitrulline, glycerol, palmitic acid, lysophosphatidylcholine (LysoPC), and lysophosphatidylethanolamine (LysoPE) before freezing, conferring cells resistance in freezing. After freezing, relative high level of proline, lysine, and glycerol maintained by SNF1 overexpression with increased content of LysoPC and LysoPE.
\end{abstract}

Conclusions: This study will increase the knowledge of the cellular response of baker's yeast cells to freezing and provide new opportunities for the breeding of low-temperature resistant strains.

Keyword: Transcriptome, Metabolome, Saccharomyces cerevisiae, Freezing stress, SNF1

\section{Background}

Frozen dough technology, which separates the processes of dough production and baking, can break through the constraints of the traditional production method of pasta products with regard to the processing time and cost and can ensure the freshness and stability of pasta products [1]. However, freezing and thawing can damage baker's yeast (Saccharomyces cerevisiae) cell activity and weaken

*Correspondence: linxiaoxuelx@163.com

${ }^{1}$ College of Food Science and Engineering, Hainan University, Haikou 570228, People's Republic of China

Full list of author information is available at the end of the article its fermentation ability, negatively affecting the quality of final pasta products and limiting the development and application of frozen dough technology [2]. The differential expression of genes brings about metabolite changes and phenotypic diversity of cells. Yeast cells adapt to stress or changing environmental conditions by initiating special procedures for gene expression [3-6] and metabolic regulation [7-9], which promote stress protection, dynamic balance and survival. However, the information about the response of yeast to freezing stress remains limited.

Snf1, a remarkably conserved serine/threonine protein kinase in eukaryotes, participates in many cellular 
activities, such as the cell cycle, proliferation, endocytosis, metabolism, and stress [10]. The Snf1 protein kinase is a complex that contains an alpha catalytic subunit Snf1, a gamma regulatory subunit Snf4, and one of three alternative beta regulatory subunits Sip1, Sip2, or Gal83 [11, 12]. Previous studies have shown that the Snf1 catalytic subunit plays a positive role in the response to various environmental stresses, such as heat shock [13], alkaline $\mathrm{pH}[14], \mathrm{Na}^{+}, \mathrm{Li}^{+}$, hygromycin $\mathrm{B}$ [15], oxidative [16], and genotoxic stresses [17]. Our previous work also showed that overexpression of $S N F 1$ was effective to improve the $S$. cerevisiae cell resistance to ethanol and high glucose stresses [18]. In contrast, Snf1 serves as a negative regulator in the ER stress response [19]. However, the role of the Snf1 catalytic subunit of baker's yeast in freezing is unclear.

In this study, to investigate the role of Snf1 in the resistance of baker's yeast cells to freezing stress, the cell survival rate and dough leavening abilities of the transformant $\mathrm{ABY}+\mathrm{S}$, which carried the overexpressed SNF1 gene, and the parental strain were tested. Furthermore, the global transcriptional and metabolic landscapes of the transformant and the parental strain were compared using transcriptome and metabonomics, respectively, before and after freezing.

\section{Results}

\section{SNF1 gene expression and growth properties}

In this study, the transformant $\mathrm{ABY}+\mathrm{S}$, which carried the overexpressed $S N F 1$, was constructed. Compared to the parental strain ABY3, the mRNA expression level of SNF1 was upregulated by 45 -fold in the transformant $\mathrm{ABY}+\mathrm{S}$ (Fig. 1). The transformant $\mathrm{ABY}+\mathrm{YP}$, which carried the plasmid Yep-PK without target genes was used as a blank control to demonstrate any possible effects of an empty vector and showed similar results to the parental strain.

Growth characteristics are essential to the application of industrial strains. To explore the impact of overexpression of SNF1 in the general growth properties of baker's yeast cells, the growth curves of the transformants and the parental strain were monitored in YEPD medium. As shown in Fig. 2, no obvious changes were displayed between the transformants and the parental strain. These results suggest that increased SNF1 gene dosage did not affect the growth properties of the baker's yeast strain used in this work under general conditions ( $2 \%$ glucose).

\section{Stress tolerance and leavening ability}

To evaluate the effect of SNF1 overexpression of baker's yeast cells in freezing stress, the cell survival rate and leavening ability were measured. As shown in Fig. 3, compared to the parental strain ABY3, the cell survival

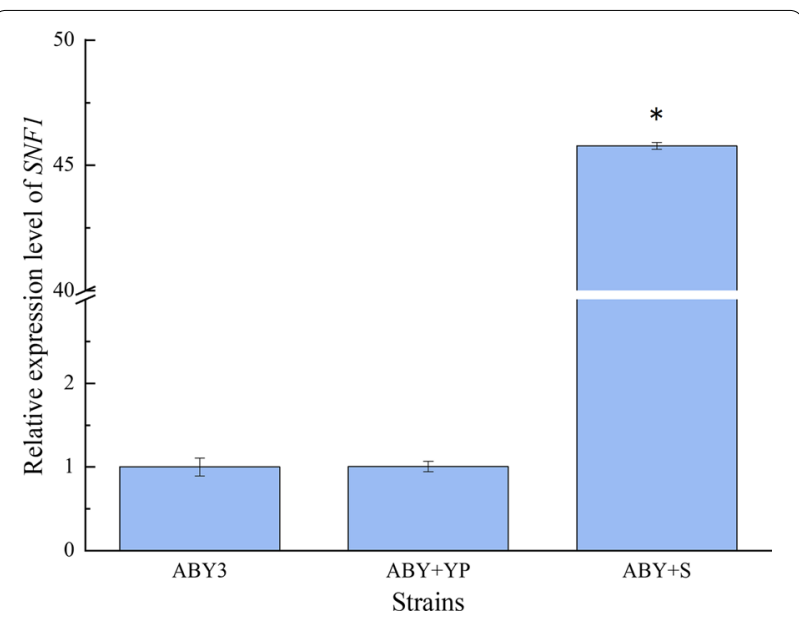

Fig. 1 mRNA level of the SNF1 gene. ABY3: the parental strain; $A B Y+Y P$ : the strain carrying the vector Yep-PK used as a blank control to demonstrate any possible effect of the empty vector; $A B Y+S$ : the transformant carrying SNF1 overexpression. Significant differences among the three strains $(A B Y 3, A B Y+Y P$, and $A B Y+S)$ were confirmed at ${ }^{*} p<0.05$

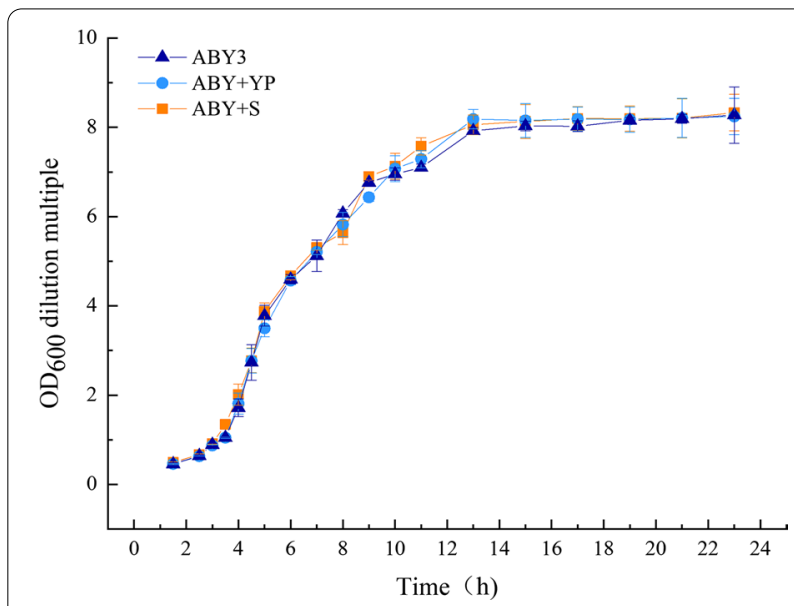

Fig. 2 Growth curves of the strains. Growth curves were monitored by measuring the cell density (OD600) at appropriate time intervals. $A B Y 3$ : the parental strain; $A B Y+Y P$ : the strain carrying the vector Yep-PK used as a blank control to demonstrate any possible effect of the empty vector; $A B Y+S$ : the transformant carrying SNF1 overexpression

rate of the transformant $\mathrm{ABY}+\mathrm{S}$ was $32 \%$ higher than that of the parental strain after 7 days of freezing. Similar behaviour was observed in the control transformant $\mathrm{ABY}+\mathrm{YP}$ to the parental strain. For the leavening ability, slight changes were observed in the transformant $\mathrm{ABY}+\mathrm{S}$ and the parental strain ABY3 before freezing (Fig. 4a, c). Nevertheless, the $\mathrm{CO}_{2}$ production in $120 \mathrm{~min}$ and leavening ability of the transformant $\mathrm{ABY}+\mathrm{S}$ were $26 \%$ and $28 \%$ higher than that of the parental strain, respectively, 


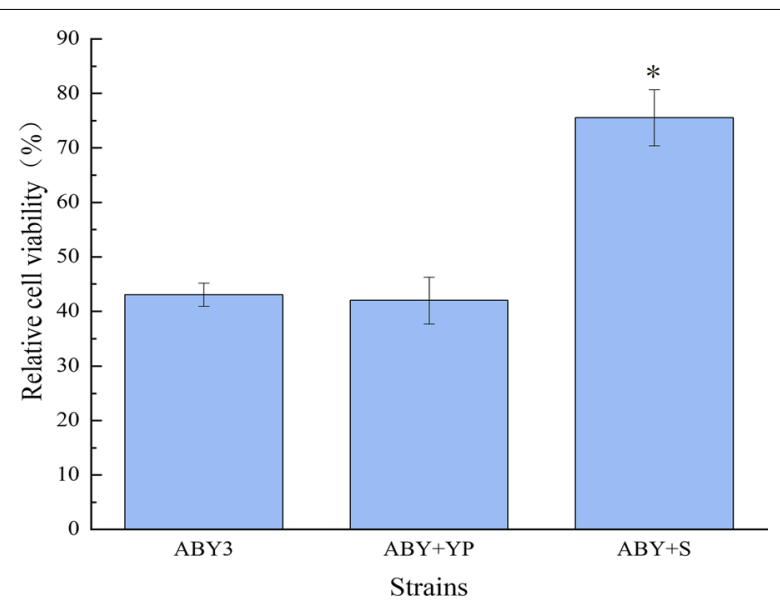

Fig. 3 Analysis of freezing tolerance. The cells were cultivated in the LSMLD medium at $-20^{\circ} \mathrm{C}$ for 7 days. ABY3: the parental strain; ABY +YP: the strain carrying the vector Yep-PK used as a blank control to demonstrate any possible effect of the empty vector; $A B Y+S$ : the transformant carrying SNF1 overexpression. Significant differences among the three strains $(A B Y 3, A B Y+Y P$, and $A B Y+S)$ were confirmed at ${ }^{*} p<0.05$

after freezing (Fig. 4b, c), and the relative leavening ability increased by $11 \%$ (Fig. $4 \mathrm{~d}$ ). The control transformant $\mathrm{ABY}+\mathrm{YP}$ displayed no significant differences compared to the parental strain ABY3. Due to SNF1 deficiency, yeast cell growth is limited under nutrient limitation [10]. In this study, overexpression of SNF1 increased the cell resistance and improved the fermentation properties of baker's yeast in freezing, but did not affect the growth in $2 \%$. These findings confirm the importance of Snf1 in stress and unstress conditions.

\section{Global gene expression}

To explore the effect of SNF1 overexpression on the global gene expression of baker's yeast during freezing, RNA-seq and bioinformatic analysis were conducted. The differentially expressed genes (DEGs) of the strains before and after freezing were analysed by cluster analysis of differential expression patterns among the four strains (the parental strains and the transformants $\mathrm{ABY}+\mathrm{S}$ non-suffered and suffered freezing). As shown in Fig. 5a, compared to the parental strain ABY3, the DEGs of the transformant $\mathrm{ABY}+\mathrm{S}$ were generally upregulated before and after freezing. The k-means clustering of all DEGs indicated that the strains were obviously divided into two groups, and the gene expression pattern of the transformant $\mathrm{ABY}+\mathrm{S}$ was significantly different from that of the parental strain ABY3. According to Fig. 5b, 1799 genes showed significant differences between the transformant $\mathrm{ABY}+\mathrm{S}$ and the parental strain $\mathrm{ABY} 3$ before freezing, of which 1453 genes were upregulated and 346 downregulated. With the same analysis method, as shown in Fig. 5c, 2308 genes were significantly different between the strains after freezing (namely, FABY $+\mathrm{S}$ and FABY3), of which 2035 genes were upregulated and 273 genes downregulated.

The KEGG enrichment analysis of the significantly differentially expressed genes of the strains before and after freezing showed that overexpression of SNF1 had the greatest effect on the proteasome pathway, especially after freezing (Fig. $5 \mathrm{~d}$, e). The function of the proteasome involves the cell cycle, DNA repair, apoptosis, gene transcription, stress response, and signal transduction [20]. When cells respond to stress, proteasomes are responsible for the degradation of intracellular misfolded proteins labelled by heat shock proteins, which directly affects the renewal of misfolded proteins and ensures related biological function [21]. In addition, the DEGs enrichment pathways before freezing included spliceosome and RNA transport; ribosome biogenesis in eukaryotes, RNA degradation, endoplasmic reticulum protein processing, RNA transport, mRNA surveillance pathway, and spliceosome were included in the DEGs enrichment pathways after freezing. Many gene transcriptional regulation and protein processing and regulatory pathways were affected by $S N F 1$ overexpression. The peroxidase pathway of the transformant $\mathrm{ABY}+\mathrm{S}$ significantly differed from that of the parental strain ABY3 after freezing. When yeast is subjected to freezing-thawing stress, the metabolism of free radicals in cells is destroyed. The accumulation of free radicals can cause or aggravate lipid peroxidation in cells, resulting in damage to the membrane system and macromolecules in the body. The regulation of the peroxidase pathway, which is related to the regulation of cell stress response, can alleviate this damage [22, 23].

The frozen survival rate and fermentation performance embody the ability of baker's yeast cells to self-protect and remain vivacious. The metabolic pathways related to cell self-protection and nutrient consumption were screened from the KEGG enrichment pathway before and after freezing, including carbon metabolism, biosynthesis of amino acids, glycolysis/gluconeogenesis, pentose phosphate pathway, glycerolipid metabolism, glycerophospholipid metabolism, and fatty acid metabolism. The differences in the expression of DEGs related to the above metabolic pathways before and after freezing were analysed (Fig. 5f, g). Carbon metabolism is the most important process of biological growth and metabolism and is strictly regulated by environmental signals. Varying degrees of cell pathological changes and death can occur as a result of carbon metabolism disorders [24]. Like most eukaryotes, the carbon metabolism of baker's yeast mainly includes glycolysis/gluconeogenesis, 

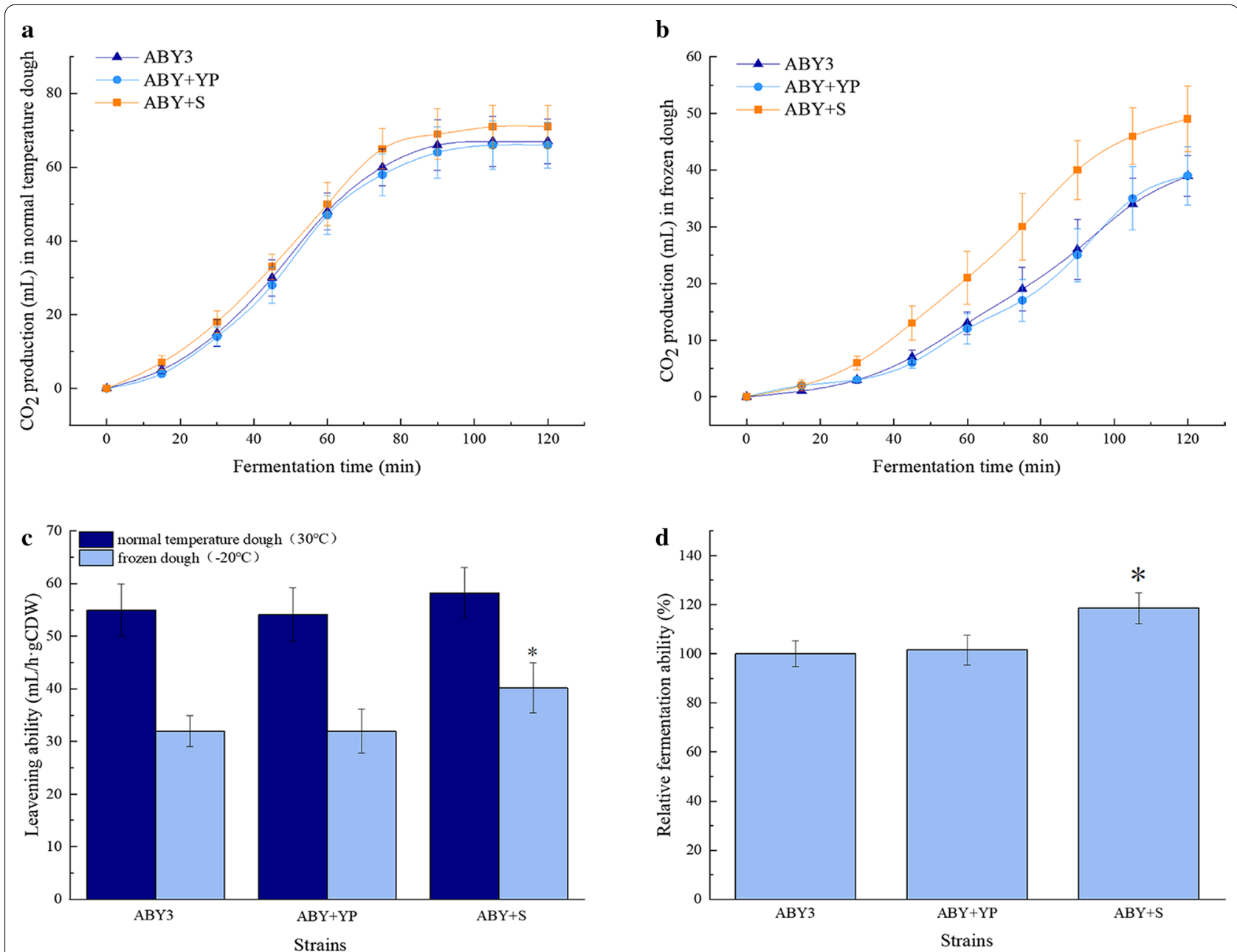

Fig. 4 Leavening ability of the strains. $\mathbf{a} \mathrm{CO}_{2}$ production in lean dough before freezing. $\mathbf{b} \mathrm{CO}_{2}$ production in lean dough after freeze-thawing at $-20^{\circ} \mathrm{C}$ for 7 days. $\mathbf{c}$ Leavening ability was determined by the millilitre of increased volume per hour per gram (dry weight) of yeast cells. $\mathbf{d}$ Relative fermentation ability was determined by the ratio of leavening ability after freeze-thawing to that before stress, and the results were expressed relative to that of the parental strain ABY3 (defined as 100\%). ABY3: the parental strain; ABY+YP: the strain carrying the vector Yep-PK used as a blank control to demonstrate any possible effect of the empty vector; ABY+S: the transformant carrying SNF1 overexpression. Significant differences among the three strains $(A B Y 3, A B Y+Y P$, and $A B Y+S)$ were confirmed at ${ }^{*} p<0.05$

TCA cycle, the pentose phosphate pathway, and the glyoxylate cycle, which directly affect the fermentation ability of strains. According to the statistics of the pathway database, 115 genes were annotated in the carbon metabolism pathway. In the pre-fermentation phase before freezing, 38 DEGs in this pathway were significantly different between the transformant $A B Y+S$ and the parental strain ABY3, of which 24 DEGs were upregulated and 14 DEGs downregulated. In the carbon metabolism pathway, 24 DEGs were involved in the glycolysis pathway, of which 18 were upregulated and 6 downregulated. 11 DEGs were involved in the pentose phosphate pathway, of which 7 DEGs were upregulated and 4 downregulated. After freezing, 49 DEGs showed significant differences in carbon metabolism between the transformant $\mathrm{ABY}+\mathrm{S}$ and the parental strain $\mathrm{ABY}$, of which 35 DEGs were upregulated and 14 downregulated. Among them, 26 DEGs were involved in the glycolysis pathway, of which 20 DEGs were upregulated and 6 downregulated. 11 DEGs were involved in the pentose phosphate pathway, of which 7 DEGs were upregulated and 4 downregulated.

As the raw material for the synthesis of proteins and enzymes, intracellular amino acids are important substances for cell growth and metabolism. Some studies have shown that yeast has a preference for organic nitrogen sourced amino acids in low temperature fermentation, and the content of intracellular amino acids 


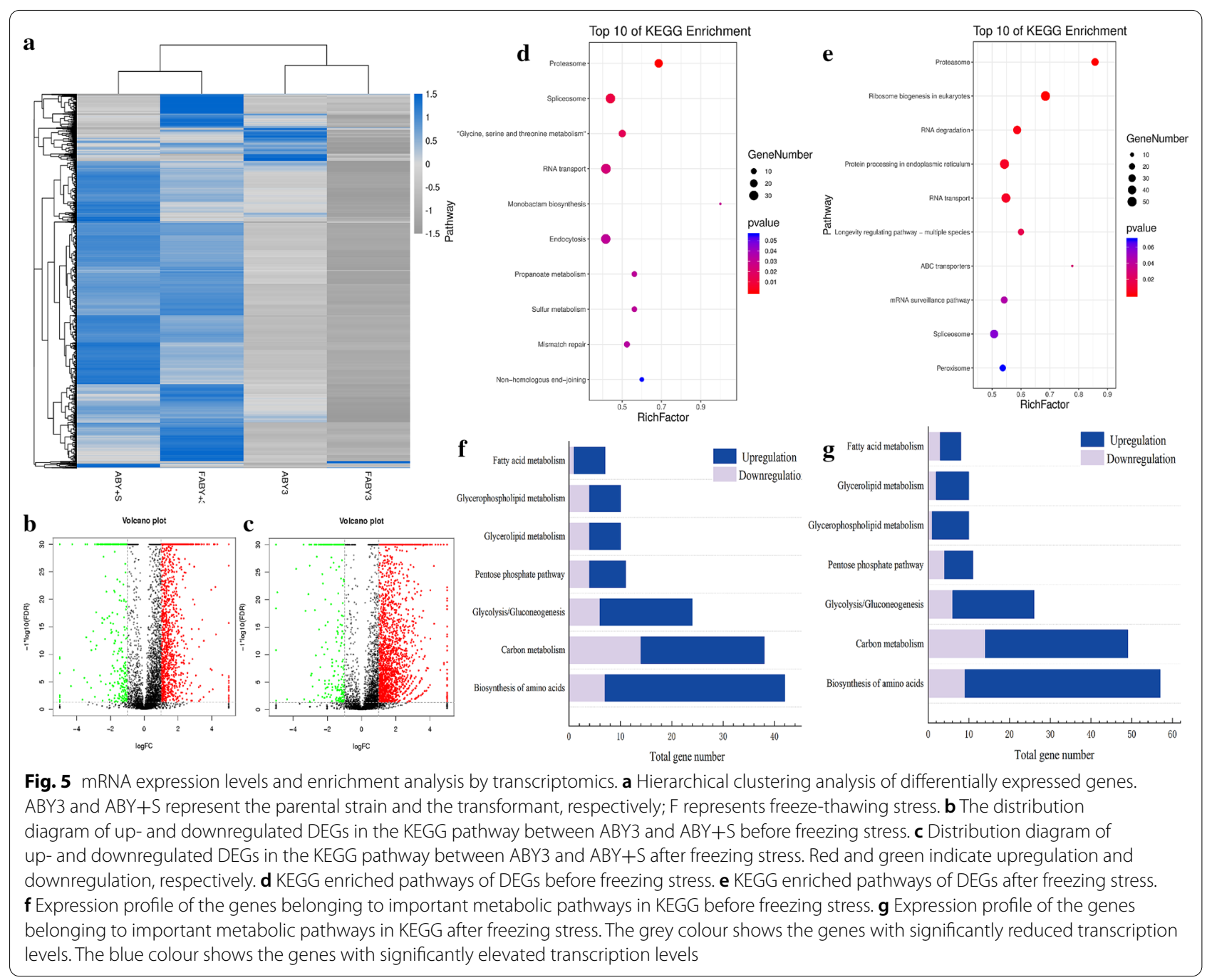

in yeast is generally downregulated after freezing stress [25-27]. Expression of the amino acid biosynthesis pathway is helpful to supplement intracellular amino acid deficiency in a low-temperature environment. According to the statistics of the pathway database, 125 genes were annotated in the amino acid biosynthesis pathway. In the pre-fermentation phase before freezing, 42 genes of the transformant $\mathrm{ABY}+\mathrm{S}$ were significantly different from the parental strain ABY3, of which 35 DEGs were upregulated and 7 DEGs downregulated, while 57 genes were significantly different in fermentation after freezing stress, of which 48 DEGs were upregulated and 9 DEGs downregulated.

The HOG-MAP kinase pathway is involved in the glycerolipid metabolism. Under freeze-thaw stress, $S$. cerevisiae activates the HOG-MAP pathway by reducing membrane fluidity. Glycerol in this pathway participates in osmotic regulation and adaptation to the low temperature growth of yeast and balances the ratio of intracellular NADH/NAD, which can be used as antifreeze [28]. According to the statistics of the pathway database, 27 genes were annotated in glycerolipid metabolism. In the pre-fermentation phase before freezing, the transformant $\mathrm{ABY}+\mathrm{S}$ showed significant differences compared to the parental strain ABY3, of which 6 DEGs were upregulated and 4 DEGs downregulated. In the fermentation phase after freezing stress, 10 genes showed significant differences, of which 8 DEGs were upregulated and 2 DEGs downregulated.

Glycerophospholipid and fatty acid metabolism are related to cell membrane composition. The plasma membrane performs a series of physiological functions in the cell, including morphological regulation and information transmission. The function of the membrane is related to the proportion of the membrane components [29]. According to the statistics of the pathway database, 38 genes were annotated in the glycerophospholipid metabolism. The transformant $\mathrm{ABY}+\mathrm{S}$ was compared with 
the parental strain $\mathrm{ABY} 3$ in the pre-fermentation phase before freezing, of which 6 DEGs were upregulated and 4 DEGs downregulated. In the fermentation phase after freezing stress, 10 genes showed significant differences, of which 9 DEGs were upregulated and 1 DEG downregulated. Overall, 21 genes were annotated in fatty acid metabolism when comparing the transformant $\mathrm{ABY}+\mathrm{S}$ with the parental strain ABY3 in the pre-fermentation phase before freezing, including 6 upregulated DEGs and 1 downregulated DEG. 8 genes showed significant differences in fermentation after freezing stress, of which 5 DEGs were upregulated and 3 DEGs downregulated.

\section{Metabolomic profiling}

In total, 77 metabolites were detected and identified by metabolic analysis in four strains (the parental strains and the transformants $\mathrm{ABY}+\mathrm{S}$ non-suffered and suffered freezing). PCA result showed that significant differences and repeatability were existed between samples (Fig. 6a). Among them, there were 17 significant differential metabolites in the pre-fermentation before freezing, of which 10 metabolites were upregulated and 7 metabolites downregulated. There were 18 significantly differential metabolites in fermentation after freezing stress, of which 3 metabolites were upregulated and 15 metabolites downregulated.

Compared to the parental strain ABY3, 17 significantly different metabolites were observed in the transformant $\mathrm{ABY}+\mathrm{S}$, of which 10 metabolites were upregulated and 7 metabolites downregulated in the pre-fermentation phase before freezing (Fig. 6b). 6 kinds of amino acids included in the 10 upregulated metabolites, among which arginine and proline have clear cryoprotective effects $[24,30]$. Related studies have shown that the fermentation ability of proline-accumulating baker's yeast in frozen dough is higher than that of the wild strain [31]. The content of glycerol, a well-known metabolite as a cell cryoprotectant [32, 33], was significantly upregulated in the transformant $\mathrm{ABY}+\mathrm{S}$. In addition, three substances in the differential metabolites were related to cell membrane components: upregulated palmitic acid and downregulated sn-1LysoPC (16:0) and sn-1LysoPE (18:1). As the protective barrier of cells, the cell membrane can maintain cell morphology through lipid changes during environmental adaptation and regulate signal transduction and stress response [28, 34-36]. In the fermentation phase after freezing stress (Fig. 6c), compared to the parental strain $A B Y 3$, there were 21 significantly different metabolites in the transformant $\mathrm{ABY}+\mathrm{S}$, of which 11 metabolites were upregulated and 10 metabolites downregulated. Protectants lysine, proline, and glycerol are included in the upregulated differential metabolites. Among the downregulated metabolites, LysoPC and
LysoPE accounted for a large proportion of the differential metabolites. LysoPC is a kind of bioactive phospholipid that is produced by phosphatidylcholine (PC) hydrolysis mediated by phospholipase in cells [29]. The effect of LysoPC on freezing tolerance of yeast has not been determined.

\section{Analysis of the enrichment pathway of significantly different genes and metabolites}

According to the transcriptome and metabonomic analyses, DEGs and differential metabolites were mainly enriched in the amino acids, glycerolipid, and glycerophospholipid metabolism (Tables 1, 2). During fermentation before freezing stress, compared to the parental strain $\mathrm{ABY} 3$, the transformant $\mathrm{ABY}+\mathrm{S}$ accumulated 6 types of amino acids including leucine, proline, serine, isoleucine, arginine, and homocitrulline, and the expression of genes related to the amino acid synthesis upregulated. The transformant $\mathrm{ABY}+\mathrm{S}$ accumulated more glycerol than the parental strain. Accordingly, the expression of gene GPP1 encoding glycerol-3-phosphatase upregulated, which regulates the synthesis of glycerol. During fermentation after freezing stress, only proline and lysine of the transformant $\mathrm{ABY}+\mathrm{S}$ exhibited more content than the parental strain. The intracellular glycerol of the transformant $\mathrm{ABY}+\mathrm{S}$ remained high with upregulated expression of GPP1 and GPP2 (encoding glycerol1-phosphatase) but downregulated expression of GUT2 (encoding glycerol-3-phosphate dehydrogenase) after freezing. The expression of genes involved in glycerol phospholipid metabolism before and after freezing was significantly different between the transformant $\mathrm{ABY}+\mathrm{S}$ and the parental strain. Simultaneously, the transformant $\mathrm{ABY}+\mathrm{S}$ and the parental strain showed difference in the accumulation of LysoPC and LysoPE.

\section{Discussion}

Snf1, a global regulator in yeast, participates in the response to stress in multiple regulatory modes. Casamayor et al. [14] showed that SNF1 mutation of Saccharomyces cerevisiae exhibited an alkali-sensitive phenotype and the role of Snf1 in the resistance to alkaline pH was largely rely on its function in the adaptation to glucose scarcity, with altered trehalose metabolism, glycogen/ glucan metabolism, sugar transport, and phosphorylation. In this work, overexpression of SNF1 increased the cell resistance and leavening ability of baker's yeast cells during freezing, which could be attributed by the altered transcriptional and metabolic patterns, but not the complete same way in the tolerance to alkalinization (Fig. 7).

Overexpression of SNF1 had an important regulatory impact in the proteasome. This finding was consistent with the results of Yao et al. [37], who showed that 


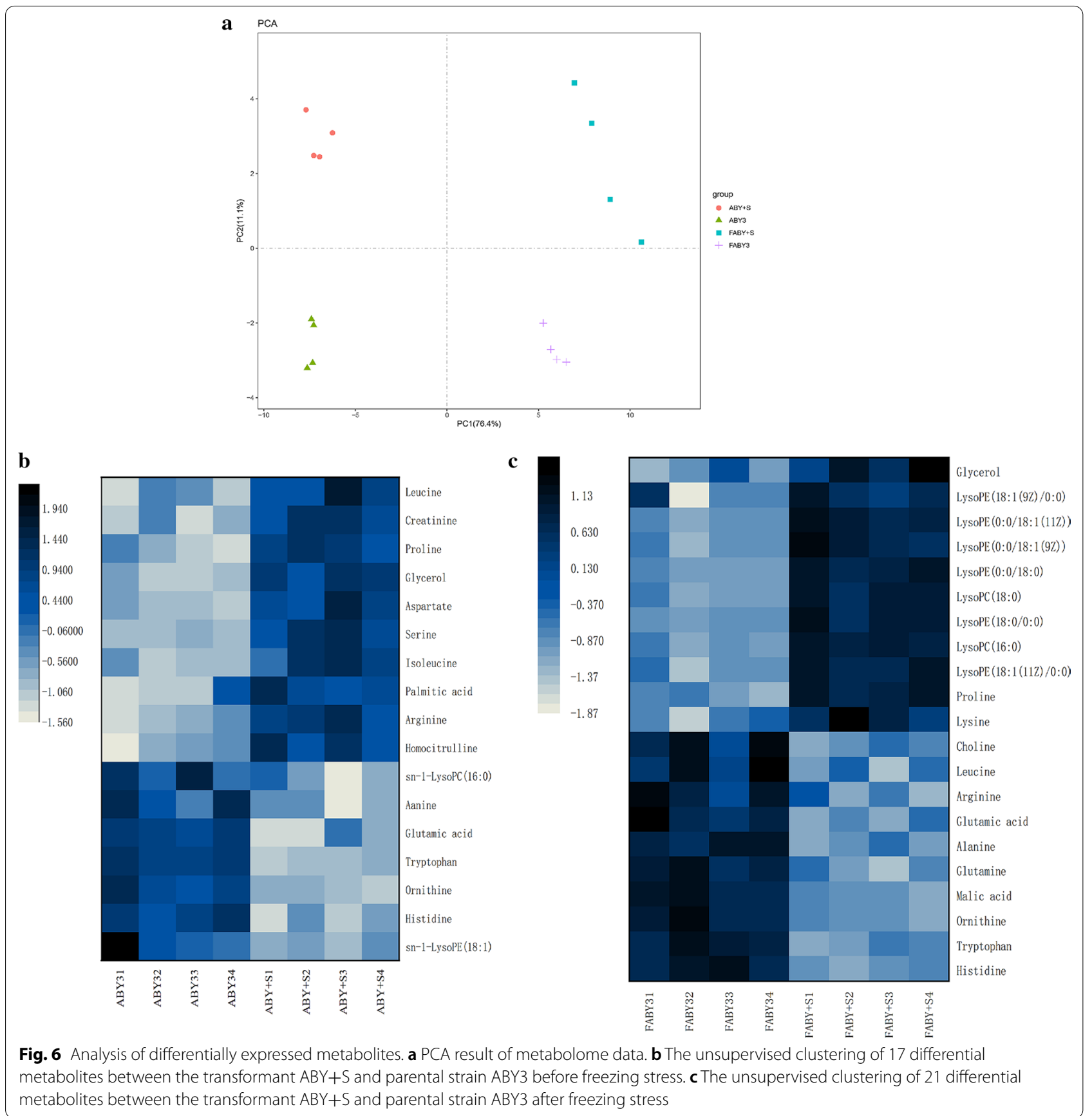

Table 1 DEGs in the enrichment pathway before freezing

\begin{tabular}{llc}
\hline Pathways & Upregulation genes & Downregulation genes \\
\hline Biosynthesis of amino acids & CDC19, TKL2, HIS7, LEU2, HIS4, CHA1, PGK1, THR4, GPM2 、 LYS20, HOM2, SAM2, & ARO7, HIS1, IRC7, YHRO33 \\
& HOM3, SER3, TRP2, MET6, LEU1, STR3 、 CYS4, TDH3, PFK1, ARG4, YHR112C, SER33, & W, ARG3, DP2, RKI1 \\
LYS12, HIS5 T TDH1, GPM1, SHM2, MET17, ILV5, PFK2, SER1, GLN1 & SCT1, DAK2, GPT2, ALD4 \\
Glycerolipid metabolism & YPR1, GPP1, DAK1, GCY1, ALE1, DGA1 & GPT2, GUT2, SCT1, PIS1 \\
Glycerophospholipid metabolism & CHO2, PSD2, SPO14, ALE1, GPD2, CKI1 & GPT \\
\hline
\end{tabular}


Table 2 DEGs in the enrichment pathway after freezing

\begin{tabular}{|c|c|c|}
\hline Pathways & Upregulation genes & Downregulation genes \\
\hline Biosynthesis of amino acids & $\begin{array}{l}\text { CYS3, CDC19, TKL2, HIS7, HIS4, CHA1, PGK1, THR4, IDP1, PRO1, SAM2, HOM3, SER3, } \\
\text { TRP2, MET6, PRS2, ARO2, STR3, NQM1, CYS4, TDH3, SER2, PFK1, ENO1, ARG4, BAT1, } \\
\text { SER33, LYS12I HIS5, TDH1, ACO2, TDH2, STR2, HOM6, TRP3, SHM2, MET17, ILV5, ILV2, } \\
\text { ERR3, LEU4, PHA2, PRS5, ARG8, ERR1, CAR1, ERR2, GLN1 }\end{array}$ & $\begin{array}{l}\text { CIT2, HIS1, ARG5,6, YHRO33 } \\
\text { W, HIS6, ARG3, IDP2, IDP3, } \\
\text { CIT3 }\end{array}$ \\
\hline Glycerolipid metabolism & ATG15, TGL2, YPR1, GPP2, GPP1, DAK1, GCY1, DGA1 & ALD4, DAK2 \\
\hline Glycerophospholipid metabolism & CDS1, CHO2, GDE1, OPI3, SPO14, CKI1, PLB2, CPT1, GPD2 & GUT2 \\
\hline
\end{tabular}

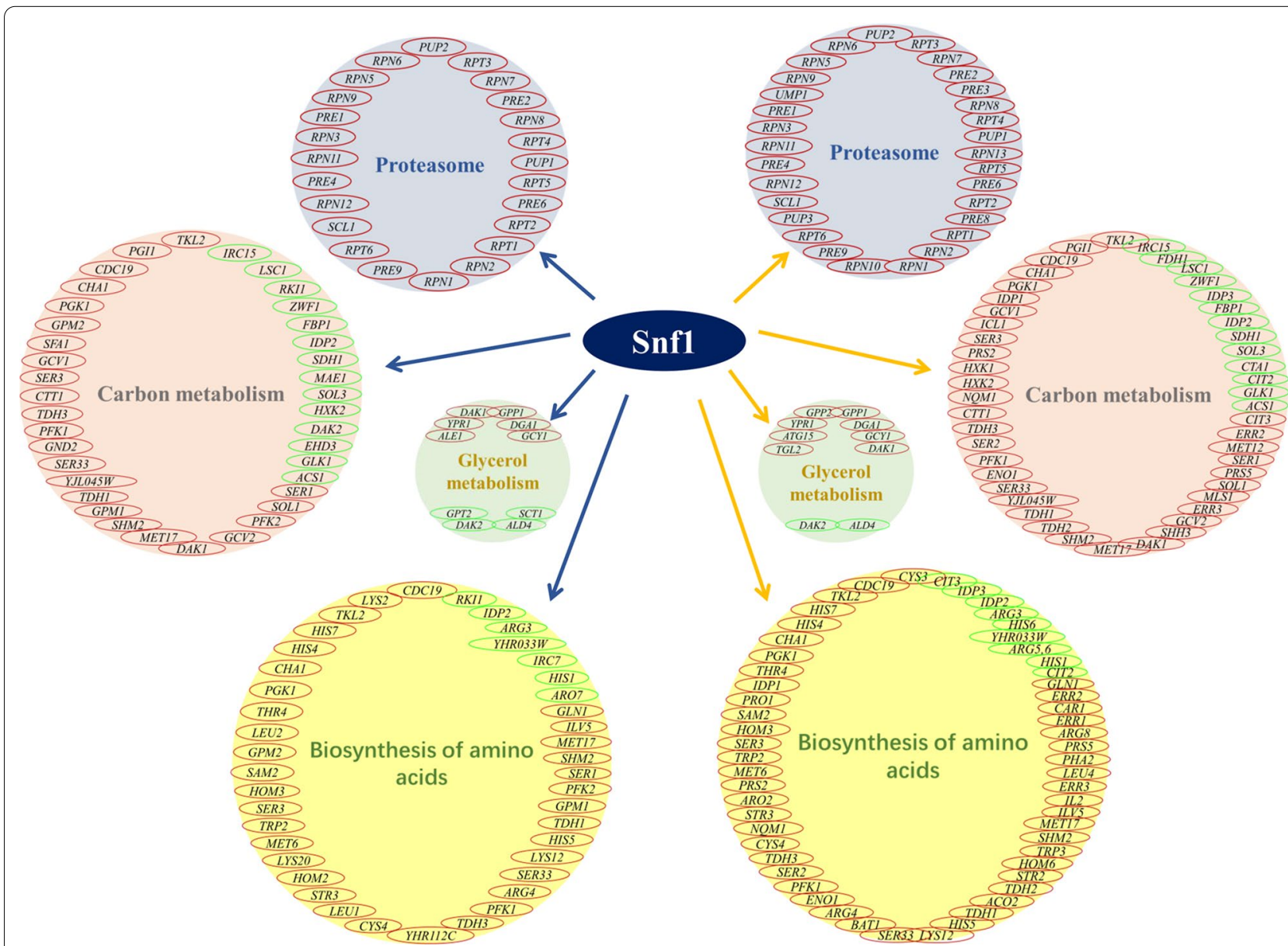

Fig. 7 Regulation of Snf1 before and after freezing. The blue arrow points before freezing; The yellow arrow points after freezing; The red and green circles indicate upregulated genes and downregulated genes, respectively

proteasomes and Snf1 interconnected in the regulation of aging. The regulatory impact was more apparent after freezing stress and was characterized by the high expression of a large number of proteasome-related genes (Additional file 1a and b). Yeast cell-controlled proteolysis via the ubiquitin-proteasome system relieves cellular stress through clearing misfolded proteins [20,21]. Snf1 regulated the expression of proteasome-related genes and enhanced the degradation efficiency of misfolded proteins under freezing stress, achieving the supervision of cell quality. This ensures that the cells still maintain high growth and metabolic activity after freezing injury.

Overexpression of SNF1 increased carbon metabolism before and after freezing with the high expression of many genes involved in carbon metabolism (Additional file $2 \mathrm{a}$ and $\mathrm{b}$ ). These findings were consistent with the results of Casamayor et al. [14], who found that Snf1 mutation downregulated the expression level of many 
genes involved in sugar metabolism of yeast induced by alkaline stress. The surviving cells maintained strong growth and metabolism abilities of baker' yeast, which ensured strong fermentation ability after freezing. Overexpression of SNF1 could effectively enhance the maltose utilization and dough leavening ability of industrial baker's yeast [38], which is also reflected after freezing stress. Snf1 regulated glycerol metabolism via the regulation of the transcription factor Adr1 in glucose [39]. In this study, SNF1 overexpression increased the accumulation of the reserve carbon source and cell protectant glycerol (Additional file $3 a$ and $b$ ). This could slow down the damage of yeast cells to a certain extent and improve the survival rate. These findings were consistent with the results of Casamayor et al. [14], suggesting that Snf1 regulated glycerol metabolism in nutrition and environmental stresses.

Before freezing, the accumulation of 6 types of amino acids in $S N F 1$ overexpression was higher than that of the wild type. The expression of genes related to the biosynthesis of amino acids before freezing was consistent with the metabonomic data (Additional file 4a). Enhanced accumulation of amino acids can improve the stress resistance of cells in coping with freezing. In addition, in the low temperature, extracellular amino acids could be consumed as organic nitrogen sources, which is likely to lead to the lack of available amino acids. The accumulation of intracellular amino acid can provide sufficient raw materials for the synthesis of proteins and enzymes in cells. Compared to the parental strain, overexpression of SNF1 decreased the accumulation of several amino acid (not including proline and lysine) after freezing. However, the transcriptional results regarding amino acid synthesis was not corresponded with the metabonomic data after freezing (Additional file 4b). Based on the metabolic pathway data, the decreased intracellular amino acids in SNF1 overexpression after freezing could be caused by the following reasons. First, enhanced carbon metabolism and glycerol synthesis could reduce the amount of carbon frame for the biosynthesis of amino acids by $S N F 1$ overexpression, weakening the synthesis of amino acids. Second, increased proteasome activity in SNF1 overexpression could improve the efficiency of misfolded protein degradation and promote the regeneration of proteins, whereas intracellular amino acids, as the raw material of protein synthesis, were consumed more in protein synthesis, resulting in a decrease in intracellular amino acids; but the correction of misfolded proteins is helpful to ensure the normal growth and metabolism of cells, maintaining a strong fermentation performance in frozen dough. The stable accumulation of proline and lysine could contribute to the high survival rate and strong leavening ability in SNF1 overexpression [40, 41].
The change of each amino acid was not the same in SNF1 overexpression under different conditions, such as that in freezing and non-freezing in this work and that in high glucose, ethanol, and heat shock stresses of our previous study [33]. In addition, Nicastro et al. [42] showed that Snf1 inhibited the biosynthesis of amino acids in amino acid-rich condition. These results exhibit no contradictory and suggest that the role of Snf1 converted in different signals. The regulation of Snf1 on the metabolism of amino acids might be in a signal-dependent manner. For example, overexpression of $S N F 1$ promoted the expression of MSN2, a transcription factor for the transport of amino acid after freezing, whereas no obvious change was observed before freezing in this work. Snf1 inhibits the translation of Gcn4, a transcription factor of amino acid synthesis in nutritional stress [43], but the regulation of Snf1 on Gcn4 in other stress is not clear. The mechanism of Snf1 regulating amino acids metabolism under different signals needs further study.

Previous studies showed that SNF1 mutation affected the phospholipid biosynthesis via the alternation of the production and proportion of fatty acids [44, 45]. In this study, overexpression of SNF1 changed the content of LysoPC and LysoPE in varied degree before and after freezing, suggesting the multiple regulation of Snf1 on phospholipid metabolism. In the metabolism of glycerophospholipids, glycerol phospholipids, including PC, phosphatidylinositol (PI), phosphatidylethanolamine (PE), phosphatidylglycerol (PG), phosphatidic acid (PA), and their corresponding lysophospholipids (LysoPC, LysoPI, LysoPE, LysoPG, LysoPA), are related to signal transduction [46]. Some studies have shown that a decrease in the ratio of PC/PE can improve S. cerevisiae cell resistance to freezing stress $[47,48]$. However, the mechanism of glycerophospholipid in the regulation of freezing response needs to be revealed.

\section{Conclusions}

Summarily, overexpression of SNF1 was effective to the improvement of the cell tolerance and leavening ability of baker's yeast in freezing, which may be related to the dynamically upregulated proteasome pathway, altered metabolism of carbon sources and protectant molecules, and changed cell membrane components. Overexpression of SNF1 could increase the content of 6 types of amino acids (including leucin, proline, serine, isoleucine, arginine, and homocitrulline), glycerol, and palmitic acid while decrease the content of LysoPC and LysoPE before freezing, probably conferring yeast cells strong resistance in freezing. After freezing, relative high level of 2 types of amino acids (including proline and lysine) and glycerol maintained by $S N F 1$ overexpression with increased content of LysoPC and LysoPE. To our knowledge, this study 
Table 3 Characteristics of strains and plasmids used in the current study

\begin{tabular}{|c|c|c|}
\hline Strains or plasmids & Relevant characteristic & Reference or source \\
\hline \multicolumn{3}{|l|}{ Strains } \\
\hline Escherichia coli DH5a & $\begin{array}{l}\text { (180 lacZ } \triangle M 15 \triangle \text { lacU169 recAl endA1 hsdR17 supE44 thi-1 gyrA } \\
\text { relA1 }\end{array}$ & $\begin{array}{l}\text { Yeast Collection Center of the Food Science and Technology } \\
\text { Laboratory of Hainan University }\end{array}$ \\
\hline S. cerevisiae $\mathrm{ABY} 3$ & Industrial baker's yeast & $\begin{array}{l}\text { Yeast Collection Center of the Food Science and Technology } \\
\text { Laboratory of Hainan University }\end{array}$ \\
\hline$A B Y+Y P$ & Yep-PK & This study \\
\hline$A B Y+S$ & Yep-PSK & This study \\
\hline \multicolumn{3}{|l|}{ Plasmids } \\
\hline pUG6 & $\begin{array}{l}\text { E. coli/S. cerevisiae shuttle vector, containing Amp }{ }^{+} \text {, loxP-KanMX- } \\
\text { loxP disruption cassette }\end{array}$ & $\begin{array}{l}\text { Yeast Collection Center of the Food Science and Technology } \\
\text { Laboratory of Hainan University }\end{array}$ \\
\hline Yep-P & $U R A 3^{+}, A m p^{R}$ ori control vector, $P G K 1_{P}-P G K 1_{T}$ & {$[18]$} \\
\hline Yep-PK & $K a n M X, P G K 1_{P}-P G K 1_{T}$ & \\
\hline Yep-PSK & KanMX,PGK1 $-S N F 1-P G K 1_{T}$ & \\
\hline
\end{tabular}

is the first attempt to investigate the impact of Snf1 in the freezing response of yeast and to explain the regulatory mechanism of Snf1 in freezing based on transcriptome and metabonomics profile. The findings will increase the knowledge of the regulatory effects of Snf1 protein kinase on the stress response of yeast. Meanwhile, the results enrich the knowledge of the cellular response of baker's yeast cells to freezing stress and can direct future biochemical and molecular studies, which could improve our understanding of the stress response of yeast.

\section{Methods}

\section{Strains and plasmids}

The genetic characteristics of strains and plasmids are shown in Table 3.

\section{Media}

E. coli was cultured in Luria-Bertani medium (consisted of $10 \mathrm{~g} / \mathrm{L}$ tryptone, $10 \mathrm{~g} / \mathrm{L} \mathrm{NaCl}$, and $5 \mathrm{~g} / \mathrm{L}$ yeast extract) at $37^{\circ} \mathrm{C}$. To select positive E. coli transformants, $100 \mu \mathrm{g} /$ $\mathrm{mL}$ ampicillin was used. The plasmid was obtained by the FastPure Plasmid Mini Kit (DC201, Vazyme, Nanjing, China).

$S$. cerevisiae was cultured in yeast extract peptone dextrose (YEPD) medium (consisted of $20 \mathrm{~g} / \mathrm{L}$ peptone, $20 \mathrm{~g} / \mathrm{L}$ glucose, and $10 \mathrm{~g} / \mathrm{L}$ yeast extract) at $30{ }^{\circ} \mathrm{C}$. To select positive $S$. cerevisiae transformants, $800 \mu \mathrm{g} / \mathrm{mL}$ G418 was used.

S. cerevisiae was statically pre-cultured in YEPD medium for $36 \mathrm{~h}$ at $30{ }^{\circ} \mathrm{C}$. Then, the first pre-cultured cells were transferred to $200 \mathrm{~mL}$ YEPD medium at a $10 \%$ inoculation amount, rotated and shaken at $30{ }^{\circ} \mathrm{C}$ for $24 \mathrm{~h}$ at $180 \mathrm{rpm}$. The second pre-cultured cells were centrifuged at $1500 \mathrm{~g}$ at $4{ }^{\circ} \mathrm{C}$ for $10 \mathrm{~min}$ and washed twice with $4{ }^{\circ} \mathrm{C}$ sterile water. To study the effect of SNF1
Table 4 Primers used in the present study

\begin{tabular}{ll}
\hline Primer & Sequence $\left(\mathbf{5}^{\prime} \boldsymbol{\rightarrow} \mathbf{3}^{\prime} \mathbf{)}\right.$ \\
\hline PGK-F & TCTAACTGATCTATCCAAAACTGA \\
PGK-R & TAACGAACGCAGAATTTC \\
K-F & CAGCTGAAGCTTCGTACGC \\
K-R & GCATAGGCCACTAGTGGATCTG \\
RT ACT1-F & ACGCTCCTCGTGCTGTCTTC \\
RT ACT1-R & GTTCTTCTGGGGCAACTCTCA \\
RT SNF1-F & CTGTCCCAGTCACCTCCAAC \\
RT SNF1-R & CTTGCCATCCTTCTTGCGTG \\
\hline
\end{tabular}

overexpression on freeze resistance, $2 \mathrm{~g}$ fresh yeast cells (70\% wet medium) were inoculated into low-sugar model liquid dough (LSMLD) medium, which was improved from the medium described by Panadero et al. [49]. The LSMLD medium was composed of $5 \mathrm{~g} / \mathrm{L}$ of glucose, $2.5 \mathrm{~g} / \mathrm{L}$ of $\left(\mathrm{NH}_{4}\right)_{2} \mathrm{SO}_{4}, 5 \mathrm{~g} / \mathrm{L}$ of urea, $16 \mathrm{~g} / \mathrm{L}$ of $\mathrm{KH}_{2} \mathrm{PO}_{4}$, $5 \mathrm{~g} / \mathrm{L}$ of $\mathrm{Na}_{2} \mathrm{HPO}_{4}, 0.6 \mathrm{~g} / \mathrm{L}$ of $\mathrm{MgSO}_{4}, 0.0225 \mathrm{~g} / \mathrm{L}$ of nicotinic acid, $0.005 \mathrm{~g} / \mathrm{L}$ of capantothenate, $0.0025 \mathrm{~g} / \mathrm{L}$ of thiamine, $0.00125 \mathrm{~g} / \mathrm{L}$ of pyridoxine, $0.001 \mathrm{~g} / \mathrm{L}$ of riboflavin, and $0.0005 \mathrm{~g} / \mathrm{L}$ of folic acid.

\section{Transformation of yeast}

The plasmids Yep-PSK and Yep-PK were transferred to the parental strain ABY3 using lithium acetate/PEG procedure according to the previous study [38]. The transformants $\mathrm{ABY}+\mathrm{S}$ and $\mathrm{ABY}+\mathrm{YP}$ were verified by $\mathrm{PCR}$ using the primers PGK-F/PGK-R and K-F/K-R shown in Table 4.

\section{Quantitative real-time polymerase chain reaction (qRT-PCR) analysis of SNF1 gene expression}

The cells were sampled from YEPD medium at $16 \mathrm{~h}$, and the expression level of SNF1 was tested according to the 
previous study [38]. The primers for the target gene SNF1 and the reference gene $A C T 1$ are shown in Table 4.

\section{Determination of growth}

S. cerevisiae cells were pre-cultured in YEPD medium at $30{ }^{\circ} \mathrm{C}$ to $\mathrm{OD}_{600}=1.20$, and transferred to $200 \mathrm{~mL}$ YEPD medium with a $4 \%$ inoculum. The cell density was measured at $30^{\circ} \mathrm{C}$ using a UV spectrophotometer (T6, Persee, Beijing, China).

\section{Determination of freeze-thaw stress tolerance}

After pre-culturation in $100 \mathrm{~mL}$ LSMLD medium for $30 \mathrm{~min}$ at $30^{\circ} \mathrm{C}, 2 \mathrm{~mL}$ of fresh $\mathrm{S}$. cerevisiae cultures were frozen at $-20^{\circ} \mathrm{C}$ for 7 days. After thawing for $30 \mathrm{~min}$ in a $30{ }^{\circ} \mathrm{C}$ water bath, the frozen suspension was diluted properly and cultured on a YEPD plate for 2 days. The percentage of colony number after stress relative to that before stress was used to determine the cell survival rate. Three independent experiments were carried out.

\section{Determination of leavening ability}

$50 \mathrm{~g}$ of mixed lean dough (consisted of $140 \mathrm{~g}$ standard flour, $72.5 \mathrm{~mL}$ of water, $4.5 \mathrm{~g}$ of fresh yeast, and $2 \mathrm{~g}$ of salt) was weighed into a $250 \mathrm{~mL}$ graduated cylinder and prefermented at $30^{\circ} \mathrm{C}$ for $30 \mathrm{~min}$, then stored at $-20^{\circ} \mathrm{C}$ for 7 days. After thawing at $30{ }^{\circ} \mathrm{C}$ for $30 \mathrm{~min}, \mathrm{CO}_{2}$ amounts were recorded at $30{ }^{\circ} \mathrm{C}$ for $120 \mathrm{~min}$. Three independent experiments were carried out.

\section{Transcriptome analysis}

The cells $(4 \mathrm{mg})$ were cultured in LSMLD medium for $30 \mathrm{~min}$ and sampled before and after freeze-thawing for transcriptome and metabonomics analyses.

Total RNA was extracted using the TRIzol method and mRNA was enriched using Oligo (dT) beads. The preparation of sequencing libraries and sequencing were conducted by Gene Denovo Biotechnology Co., Ltd (Guangzhou, China). One experiment was carried out.

After filtering the off-machine data to get clean and high-quality data, the reads were compared to the reference genome, and the transcripts were assembled by Cufflinks to obtain the known transcripts and new transcripts. Gene abundances were quantified by RSEM software [50]. The gene expression level was normalized using the Fragments Per Kilobase of transcript per Million fragments mapped (FPKM). The DEGs were identified using the edge $R$ package, and a fold change $\geq 2$ and a false discovery rate $(\mathrm{FDR})<0.05$ was considered to be a significant difference. The functional enrichment of the DEGs was analysed.

\section{Metabonomic analysis}

The metabolites were extracted according to Yuan et al. [51]. Untargeted UPLC-QTOF-MS/MS was conducted according to Shu et al. [52] by LipidALL Technologies Co., Ltd (Changzhou, China). Four biological replicates were performed independently.

Data processing was performed according to Shu et al. [52]. The unknown differential metabolites were qualitatively identified by the comparison with standards of HMDB, METLIN, and other database, and $p$ $<0.05$ in the one-way analysis of variance (ANOVA) and a fold change $>1.5$ were considered to be the significant difference.

\section{Statistical analysis}

ANOVA and Least-Significant Difference (LSD) tests were used to determine the difference between the transformants and the parental strain in Figs 1, 2, 3, 4, and $p<0.05$ was considered as significantly different.

\section{Supplementary Information}

The online version contains supplementary material available at https://doi. org/10.1186/s12934-020-01503-0.

Additional file 1. Proteasome pathway information related to freezing stress. Differential expression between the SNF1 overexpression transformant and the parental strain in proteasome (a) before freezing stress and (b) after freezing stress. The red and green arrows indicate upregulation and downregulation, respectively.

Additional file 2. Carbon metabolism pathway information related to freezing stress. Differential expression between the SNF1 overexpression transformant and the parental strain in carbon metabolism (a) before freezing stress and (b) after freezing stress. The red and green arrows indicate upregulation and downregulation, respectively.

Additional file 3. Glycerol metabolism pathway information related to freezing stress. Differential expression between the SNF1 overexpression transformant and the parental strain in glycerol metabolism (a) before freezing stress and (b) after freezing stress. The red and green arrows indicate upregulation and downregulation, respectively.

Additional file 4. Amino acid biosynthesis pathway information related to freezing stress. Differential expression between the SNF1 overexpression transformant and the parental strain in amino acid biosynthesis (a) before freezing stress and (b) after freezing stress. The red and green arrows indicate upregulation and downregulation, respectively.

\section{Authors' contributions}

$X \mathrm{~L}$ conceived and designed the research. $L M$ performed the experiments and drafted the manuscript. XY and HYJ performed fermentation experiments. $\mathrm{XPH}$ and SXL revised the manuscript. All authors read and approved the final manuscript.

\section{Funding}

This study was financially supported by the Scientific Research Foundation of Hainan University (grant number KYQD1660) and the Foundation (No. 2018KF001) of Key Laboratory of Industrial Fermentation Microbiology of Ministry of Education and Tianjin Key Lab of Industrial Microbiology (Tianjin University of Science \& Technology). 


\section{Availability of data and materials}

All data generated or analysed during this study are included in this published article. The transcriptomics data are presented at https://www.ncbi.nlm.nih. gov/Traces/study/?acc=PRJNA681749. The metabolomics data are presented at https://data.mendeley.com/datasets/6jw4fnrcsm/1.

\section{Ethics approval and consent to participate}

Not applicable.

\section{Consent for publication}

Not applicable.

\section{Competing interests}

The authors declare that they have no competing interests.

\section{Author details}

${ }^{1}$ College of Food Science and Engineering, Hainan University, Haikou 570228, People's Republic of China. ${ }^{2}$ Engineering Research Center of Utilization of Tropical Polysaccharide Resources, Ministry of Education, Haikou 570228, People's Republic of China. ${ }^{3}$ Hainan Key Laboratory of Food Nutrition and Functional Food, Haikou 570228, People's Republic of China. ${ }^{4}$ College of Science, Hainan University, Haikou 570228, People's Republic of China.

Received: 13 October 2020 Accepted: 26 December 2020

Published online: 07 January 2021

\section{References}

1. Wang $P$, Jin ZY, Xu XM. Physicochemical alterations of wheat gluten proteins upon dough formation and frozen storage- A review from gluten, glutenin and gliadin perspectives. Trends Food Sci Tech. 2015;46:189-98.

2. Ayati S-V, Hamdami N, Le-Bail A. Frozen Sangak dough and bread properties: impact of pre-fermentation and freezing rate. Int J Food Prop. 2017:20:782-91

3. Nuño-Cabanes C, Ugidos M, Tarazona S, Martín-Expósito M, Conesa A. A multi-omics dataset of heat-shock response in the yeast RNA binding protein Mip6. Sci Data. 2020;7:69.

4. García R, Pulido V, Orellana-Muñoz S, Nombela C, Arroyo J. Signalling through the yeast MAPK Cell Wall Integrity pathway controls P-body assembly upon cell wall stress. Sci Rep. 2019;9:3186.

5. Khoa T, Yogita J, Deepika J, Green EM. Set4 is a chromatin-associated protein, promotes survival during oxidative stress, and regulates stress response genes in yeast. J Biol Chem. 2018;293:14429-43.

6. Yabuki Y, Ikeda A, Araki M, Kajiwara K, Mizuta K, Funato K. Sphingolipid/ Pkh1/2-TORC1/Sch9 signaling regulates ribosome 2 biogenesis in tunicamycin-induced stress response in yeast. Genetics. 2019. https://doi. org/10.1534/genetics.118.301874.

7. LV YJ, Wang X, Ma Q, Bai X, Li BZ, Zhang WW, Yuan YJ. Proteomic analysis reveals complex metabolic regulation in Saccharomyces cerevisiae cells against multiple inhibitors stress. Appl Microbiol Biot. 2014;98:2207-21.

8. Bubis JA, Spasskaya DS, Gorshkov VA, Kjeldsen F, Kofanova AM, Lekanov DS, Gorshkov MV, Karpov VL, Tarasova IA, Karpov DS. Rpn4 and proteasome-mediated yeast resistance to ethanol includes regulation of autophagy. Appl Microbiol Biot. 2020;104:4027-41.

9. Randez-Gil F, Córcoles-Saez I, Estruch F, Prieto JA. Lipid metabolism regulation and its relationship with cold stress response in yeast. Cryobiology. 2018:85:127.

10. Coccetti P, Nicastro R, Tripodi F. Conventional and emerging roles of the energy sensor Snf1/AMPK in Saccharomyces cerevisiae. Microb Cell. 2018:5:482-94

11. Hardie DG, Carling D, Carlson M. The AMP-activated/SNF1 protein kinase subfamily: metabolic sensors of the eukaryotic cell? Annu Rev Biochem. 1998;67:821-55.

12. Hedbacker K, Carlson M. SNF1/AMPK pathways in yeast. Front Biosci. 2008;13:2408-20.

13. Hahn JS, Thiele DJ. Activation of the Saccharomyces cerevisiae heat shock transcription factor under glucose starvation conditions by Snf1 protein kinase. J Biol Chem. 2004;279:5169-76.
14. Casamayor A, Serrano R, Platara M, Casado C, Ruiz A, Arino J. The role of the Snf1 kinase in the adaptive response of Saccharomyces cerevisiae to alkaline pH stress. Biochem J. 2012;444:39-49.

15. Hong SP, Carlson M. Regulation of Snf1 protein kinase in response to environmental stress. J Biol Chem. 2007:282:16838-45.

16. Pereira C, Pereira AT, Costa V. Activation of SNF1/AMPK mediates the mitochondrial derepression, resistance to oxidative stress and increased lifespan of cells lacking the phosphatase Sit4p. BBA-Mol Cell Res. 2020;1867:118660.

17. Bonomelli B, Martegani E, Colombo S. Lack of SNF1 induces localization of active Ras in mitochondria and triggers apoptosis in the yeast Saccharomyces cerevisiae. Biochem Bioph Res Co. 2020;523:130-4.

18. Mizuno T, Masuda Y, Irie K. The Saccharomyces cerevisiae AMPK, Snf1, negatively regulates the Hog1 MAPK pathway in ER stress response. Plos Genet. 2015;11:e1005491.

19. Hirano H, Kimura Y, Kimura A. Biological significance of co- and posttranslational modifications of the yeast $26 \mathrm{~S}$ proteasome. J Proteomics. 2016;134:37-46.

20. Kimura A, Kurata Y, Nakabayashi J, Kagawa H, Hirano H. N-Myristoylation of the Rpt2 subunit of the yeast 265 proteasome is implicated in the subcellular compartment-specific protein quality control system. J Proteomics. 2016;130:33-41.

21. Cai GH, Wang GD, Wang L, Liu Y, Pan JW, Li DQ. A maize mitogenactivated protein kinase kinase, ZmMKK1, positively regulated the salt and drought tolerance in transgenic Arabidopsis. J Plant Physiol. 2014;171:1003-16.

22. Huang XS, Wang W, Zhang Q, Liu JH. A basic Helix-Loop-Helix transcription factor, PtrbHLH, of Poncirus trifoliata confers cold tolerance and modulates peroxidase-mediated scavenging of hydrogen peroxide. Plant Physiol. 2013;162:1178-94.

23. Tripodi F, Nicastro R, Reghellin V, Coccetti P. Post-translational modifications on yeast carbon metabolism: Regulatory mechanisms beyond transcriptional control. BBA-Gen Subjects. 2015;1850:620-7.

24. Beltran G, Rozès N, Mas A, Guillamón JM. Effect of low-temperature fermentation on yeast nitrogen metabolism. World J Microb Biot. 2006;23:809-15.

25. Zhao XR, Zou HJ, Fu JW, Chen J, Zhou JW, Du GC. Nitrogen regulation involved in the accumulation of urea in Saccharomyces cerevisiae. Yeast. 2013;30:437-47.

26. Fayyad-Kazan M, Feller A, Bodo E, Boeckstaens M, Marini AM, Dubois $\mathrm{E}$, Georis I. Yeast nitrogen catabolite repression is sustained by signals distinct from glutamine and glutamate reservoirs. Mol Microbiol. 2016;99:360-79.

27. Jin Y, Weining S, Nevo E. A MAPK gene from Dead Sea fungus confers stress tolerance to lithium salt and freezing-thawing: Prospects for saline agriculture. P Natl Acad Sci USA. 2005;102:18992-7.

28. Woodman BS, Trousdale C, Conover J, Kim K. Yeast membrane lipid imbalance leads to trafficking defects toward the Golgi: lipid affects membrane trafficking. Cell Biol Int. 2018;42:890-902.

29. Cheng YF, Du ZL, Zhu H, Guo XN, He XP. Protective effects of arginine on Saccharomyces cerevisiae against ethanol stress. Sci Rep. 2016;6:31311.

30. Shi XJ, Miao YL, Chen JY, Chen J, Wang JN. The relationship of freeze tolerance with intracellular compounds in baker's yeasts. Appl Biochem Biotech. 2014;172:3042-53.

31. Aslankoohi E, Rezaei MN, Vervoort Y, Courtin CM, Verstrepen KJ. Glycerol production by fermenting yeast cells is essential for optimal bread dough fermentation. PLOS ONE. 2015;10:13.

32. Turk M, Gostintar C. Glycerol metabolism genes in Aureobasidium pullulans and Aureobasidium subglaciale. Fungal Biol. 2018;122:63-73.

33. Meng L, Liu HL, Lin X, Hu XP, Teng KR, Liu SX. Enhanced multi-stress tolerance and glucose utilization of Saccharomyces cerevisiae by overexpression of the SNF1 gene and varied beta isoform of Snf1 dominates in stresses. Microb Cell Fact. 2020;19:134

34. Beltran G, Novo M, Guillamon JM, Mas A, Rozes N. Effect of fermentation temperature and culture media on the yeast lipid composition and wine volatile compounds. Int J Food Microbiol. 2008;121:169-77.

35. Beltran G, Novo M, Leberre V, Sokol S, Labourdette D, Guillamon JM, Mas A, Francois J, Rozes N. Integration of transcriptomic and metabolic analyses for understanding the global responses of low-temperature winemaking fermentations. FEMS Yeast Res. 2006;6:1167-83. 
36. Chatterjee MT, Khalawan SA, Curran BPG. Cellular lipid composition influences stress activation of the yeast general stress response element (STRE). Microbiology. 2000;146:877-84.

37. Yao Y, Tsuchiyama S, Yang C, Bulteau AL, He C, Robison B, Tsuchiya M, Miller D, Briones V, Tar K, Potrero A, Friguet B, Kennedy BK, Schmidt M. Proteasomes, Sir2, and Hxk2 form an interconnected aging network that impinges on the AMPK/Snf1-regulated transcriptional repressor Mig1. PLoS Genet. 2015;11:e1004968.

38. Zhang CY, Bai XW, Lin X, Liu XE, Xiao DG. Effects of SNF1 on maltose metabolism and leavening ability of baker's yeast in lean dough. J Food Sci. 2015;80:M2879-85.

39. Ratnakumar S, Kacherovsky N, Arms E, Young ET. Snf1 controls the activity of Adr1 through dephosphorylation of Ser230. Genetics. 2009;182:735-45.

40. Sasano Y, Haitani Y, Ohtsu I, Shima J, Takagi H. Proline accumulation in baker's yeast enhances high-sucrose stress tolerance and fermentation ability in sweet dough. Int J Food Microbiol. 2012;152:40-3.

41. Tsolmonbaatar A, Hashida K, Sugimoto Y, Watanabe D, Furukawa S, Takagi $H$. Isolation of baker's yeast mutants with proline accumulation that showed enhanced tolerance to baking-associated stresses. Int J Food Microbiol. 2016;238:233-40.

42. Nicastro R, Tripodi F, Guzzi C, Reghellin V, Khoomrung S, Capusoni C, Compagno C, Airoldi C, Nielsen J, Alberghina L, Coccetti P. Enhanced amino acid utilization sustains growth of cells lacking Snf1/AMPK. Biochim Biophys Acta. 2015;1853:1615-25.

43. Shirra MK, MCCartney RR, Zhang C, Shokat KM, Schmidt MC, Arndt KM. A chemical genomics study identifies Snf1 as a repressor of GCN4 translation. J Biol Chem. 2008;283:35889-98.

44. Shirra MK, Patton-Vogt J, Ulrich A, Liuta-Tehlivets O, Kohlwein SD, Henry SA, Arndt KM. Inhibition of acetyl coenzyme A carboxylase activity restores expression of the INO1 gene in a snf1 mutant strain of Saccharomyces cerevisiae. Mol Cell Biol. 2001;21:5710-22.

45. Hofbauer HF, Schopf FH, Schleifer H, Knittelfelder OL, Pieber B, Rechberger GN, Wolinski H, Gaspar ML, Kappe CO, Stadlmann J, Mechtler K,
Zenz A, Lohner K, Tehlivets O, Henry SA, Kohlwein SD. Regulation of gene expression through a transcriptional repressor that senses acyl-chain length in membrane phospholipids. Dev Cell. 2014;29:729-39.

46. Ayyash M, Algahmi A, Gillespie J, Oelkers P. Characterization of a lysophospholipid acyltransferase involved in membrane remodeling in Candida albicans. BBA-Mol Cell Biol L. 2014;1841:505-13.

47. Tronchoni J, Rozes N, Querol A, Guillamon JM. Lipid composition of wine strains of Saccharomyces kudriavzevii and Saccharomyces cerevisiae grown at low temperature. Int J Food Microbiol. 2012;155:191-8.

48. Redón M, Guillamón JM, Mas A, Rozès N. Effect of growth temperature on yeast lipid composition and alcoholic fermentation at low temperature. Eur Food Res Technol. 2011;232:517-27.

49. Panadero J, Randez-Gil F, Prieto JA. Validation of a flour-free model dough system for throughput studies of baker's yeast. Appl Environ Microbiol. 2005;71:1142-7.

50. Li B, Dewey CN. RSEM: accurate transcript quantification from RNA-Seq data with or without a reference genome. BMC Bioinformat. 2011;12:323.

51. Yuan M, Breitkopf SB, Yang XM, Asara JM. A positive/negative ionswitching, targeted mass spectrometry-based metabolomics platform for bodily fluids, cells, and fresh and fixed tissue. Nat Protoc. 2012;7:872-81.

52. Shu HZ, Zhang WM, Yun YH, Chen WJ, Zhong QP, Hu YY, Chen HM, Chen WX. Metabolomics study on revealing the inhibition and metabolic dysregulation in Pseudomonas fluorescens induced by 3-carene. Food Chem. 2020;329:127220.

\section{Publisher's Note}

Springer Nature remains neutral with regard to jurisdictional claims in published maps and institutional affiliations.
Ready to submit your research? Choose BMC and benefit from:

- fast, convenient online submission

- thorough peer review by experienced researchers in your field

- rapid publication on acceptance

- support for research data, including large and complex data types

- gold Open Access which fosters wider collaboration and increased citations

- maximum visibility for your research: over 100M website views per year

At BMC, research is always in progress.

Learn more biomedcentral.com/submissions 